\title{
La formation permanente des ingénieurs hydrauliciens dans les pays en voie de développement : bilan et perspectives
}

\author{
Michel Detay (*)
}

\section{Introduction}

L'Eau est le patrimoine essentiel de l'espèce humaine. A ce titre on pourrait croire que l'Homme la gère, l'économise, la préserve, mais les hommes de tous les pays ont été négligents et imprévoyants. Si l'on n'améliore pas considérablement la gestion des ressources en eau, l'avenir de l'espèce humaine et de bien d'autres espèces est compromis [1]. Aujourd'hui, plus de deux milliards d'hommes n'ont pas accès à l'eau potable, et le développement accéléré des grandes métropoles et leur multiplication ne font qu'accroître une demande mal ou pas satisfaire.

La situation de crise que connaissent les pays en voie de développement semblait jusqu'alors épargner les pays industrialisés. En quelques mois cette assurance s'est quelque peu ébranlée. Bien que nos «crises de l'eau " n'aient pas la gravité de celles qui paralysent le tiers monde il serait dangereux de les sous-estimer : les fleuves sont pollués par les nitrates, les métaux lourds, les matières organiques; les lacs et les rivières, saturés de phosphates, sont frappés d'eutrophisation; certains écosystèmes entiers sont en danger de mort, comme le lac Baïkal ; la désertification est en progression constante, même en Europe [2]. On ne doit pas seulement améliorer la gestion des ressources en eau; on doit aussi favoriser la communication, former et informer. Enfin, pour reprendre les termes du discours de clôture de M. Elie Wiesel résumant les principales recommandations de la conférence des lauréats du prix Nobel à Paris en janvier 1988: "l'éducation doit devenir une priorité absolue de tous les budgets et doit aider à valoriser tous les aspects de la créativité humaine " [3].

\section{La problématique}

La conférence de Mar del Plata (Argentine) a douze ans. La Décennie Internationale de l'Eau Potable et de l'Assainissement (DIEPA) s'achève en 1990 et pourtant défaut d'alimentation en eau et désertification n'ont jamais fait autant de victimes. La maîtrise de l'eau est l'un des thèmes majeurs de notre monde. Au seuil de l'an 2000, la gestion globale des ressources en eau dans les pays en développement englobe quatre thèmes majeurs: la ressource, le milieu rural, le milieu urbain et l'environnement.

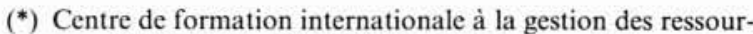
ces en eau (CEFIGRE), B.P. 113, 06561 Valbonne Cedex - France.

\section{Professional training of hydraulics engineers for developing countries : results and prospects}

After reviewing the problems of global management of water resources, under four main headings, we present in detail the activities of the International Training Centre for Water Resources Management (ITCWRM). ITCWRM's mission is analyzed together with its mode of operation and evolution since it's creation in 1977. Finally, using the results of the last decade we draw conclusion concerning ITCWRM's actions and propose recommendations for future water engineering training in low incomes countries. 


\subsection{La gestion de la ressource}

Bien qu'il n'y ait pas d'arithmétique du malheur il faut savoir que la demande en eau du tiers monde, soit 4,8 milliards de personnes (les trois quarts de la population mondiale dont $40 \%$ à moins de 15 ans), n'est correctement couverte, en qualité et en quantité, qu'à $15 \%$ [4]. Il est classiquement admis que 40000 personnes meurent chaque jour faute d'un approvisionnement en eau adéquat. Chaque année quatre millions d'enfants de moins de cinq ans meurent de maladies d'origine hydrique.

L'éducation, l'alimentation et la prévention sont les instruments essentiels d'une politique démographique et d'une réduction de la mortalité infantile. Il convient de proposer des thèmes de formation ayant trait à la gestion de la ressource, de mieux faire connaître les nouveaux outils de gestion et de faire prendre conscience de la nécessité de gérer le cycle de l'eau. En particulier, dans les pays en voie de développement, les sciences et la technologie doivent être rendues accessibles pour permettre aux populations locales de contrôler leur futur et de définir euxmêmes les connaissances nécessaires à leur devenir.

\subsection{L'alimentation en eau et l'assainissement en milieu urbain}

Depuis le début du XVIII ${ }^{\mathrm{e}}$ siècle, la population humaine a été multipliée par huit et l'espérance de vie moyenne a plus que doublé. Aujourd'hui près de cinq milliards d'habitants vivent sur la Terre. En l'an 2000, deux milliards d'hommes, de femmes et d'enfants vivront dans les métropoles du Tiers-Monde. Témoins, ces villes africaines qui ont vu leur population multipliée par sept au cours des trente dernières années. Si cette tendance persiste, elle devrait tripler dans les dix à quinze ans à venir.

Le développement urbain est un phénomène inéluctable. Dans les pays les moins développés, il se fera, pour une large part, par la création de quartier spontanés en périphérie des villes ou dans des espaces laissés libres et insalubres, espaces verts, etc... Ajoutée à celles du logement, de l'éducation et des communications, la situation de l'approvisionnement en eau et de l'assainissement d'ores et déjà tragique dans bien des cas, ne pourra que s'aggraver.

\subsection{Le développement rural}

Le milieu rural est l'un des acteurs importants de l'économie africaine. C'est la composante essentielle de l'autosuffisance alimentaire et un élément majeur du développement des échanges monétaires. La production agricole comprend la lutte contre la désertification et l'irrigation pourrait permettre d'optimiser le développement rural et de diminuer ainsi le rôle de tuteur économique tenu par les pays développés. L'homme, le cheptel et les cultures y trouveront leur moyen d'existence.

\subsection{L'environnement}

La désertification menace plus de $20 \%$ des terres émergées, soit 30 à 40 millions de $\mathrm{km}^{2}$ de zones arides ou semi-arides (depuis 1980, $200000 \mathrm{~km}^{2} /$ an sont perdus). Les conclusions de la conférence de Nairobi sur la désertification [5] prévoient que «le tiers des terres arables du monde aura cessé d'être productif dans les 50 ans à venir ».

Les tentatives pour gérer les interactions des individus et de leur environnement sont aussi vieilles que les civilisations humaines, mais les changements quantitatifs de vitesse, d'échelle et de complexité imposent des modifications qualitatives et quantitatives de notre approche [1] Nous nous devons, par le biais de la formation, d'agir et de faire agir de sorte que notre passé ne devienne pas l'avenir des pays en voie de développement.

Chacun sait que les problèmes les plus importants qu'affronte l'humanité sont aujourd'hui à la fois universels et interdépendants. L'absence de maitrise des ressources en eau coûte plusieurs millions de vies humaines par an. La formation est l'un des instruments stratégiques capables de susciter et d'accompagner les évolutions et les changements, par l'accroissement et la diversification des compétences collectives et individuelles.

\section{La création d'un centre international de gestion des ressources en eau}

Le projet de créer un organisme de formation au service de la Communauté Internationale de l'Eau, destiné à aider les nations en développement, a été annoncé lors de la $18^{\mathrm{e}}$ Conférence de l'UNESCO en 1974 ; ce projet a été présenté à Nairobi, en avril 1975. Enfin, le Centre de Formation Internationale à la Gestion des Ressources en Eau (le CEFIGRE) s'est définitivement concrétisé à l'occasion de la conférence de Mar del Plata en 1977.

Le CEFIGRE, dans sa conception est destiné aux cadres et dirigeants du secteur de l'eau des pays en voie de développement. Les sessions internationales de formation proposées abordent tant les aspects techniques qu'institutionnels, sociaux, financiers, économiques ou de gestion des ressources humaines. Enfin, elles sont conçues de manière à inciter les participants à un échange d'expériences. Depuis sa conception, qui le vouait à la formation et aux échanges, le CEFIGRE a évolué dans de nouvelles directions, affinant un peu plus sa vocation d'élément moteur de l'innovation méthodologique et technique. Il s'est adapté de ce fait aux besoins qui se sont fait jour dans des régions en transformation constante.

\subsection{Les grandes dates du CEFIGRE}

1977 : création du CEFIGRE

1979: le séminaire international organisé par le Comité Inter-africain d'Etudes Hydrauliques (CIEH) et le CEFIGRE, fixe les orientations et les missions du CEFIGRE à long terme.

1980-1982 : sept sessions de formation et d'échanges sont organisées. Elles réunissent 167 participants 
venus du continent africain, de Madagascar et d'Haïti.

1983-1984 : le CEFIGRE consolide ses acquis et développe ses activités. La montée en puissance se traduit par douze sessions internationales et deux opérations nationales. Au total, 45 pays ont été concernés, réunissant 300 participants.

1985-1986: l'expansion géographique demandée par le Conseil Scientifique International du CEFIGRE l'amène à dépasser le continent africain et le Proche Orient. L'action s'étant désormais à la Chine, à la Thaïlande et à l'Amérique Latine.

1986-1987: le CEFIGRE crée son centre de Bangkok et met en place trois conseils scientifiques régionaux pour l'Asie, l'Afrique et la Méditerranée. Les programmes nationaux se développent. Le CEFIGRE, qui a atteint sa maturité, repart dans une nouvelle phase de croissance.

1988-1989: l'innovation technologique est inscrite au programme du CEFIGRE, à commencer par l'application de l'informatique à la gestion des ressources en eau.

1990...: C'est l'année de l'internationalisation du Conseil d'Adminstration du CEFIGRE.

\subsection{Les produits du CEFIGRE}

A la demande de ses partenaires, pays, organisations régionales ou internationales, le CEFIGRE organise des sessions internationales de formation, du conseil et de l'ingénierie de formation, enfin, le Centre a une politique orientée vers l'innovation et ce particulièrement dans le domaine des technologies nouvelles et de l'informatique.

\subsubsection{Les sessions internationales de formation}

Le Centre propose chaque année un programme de formation, qui touche aux différents métiers de l'eau et s'adresse de façon prioritaire aux dirigeants et aux personnels d'encadrement (cadres supérieurs et moyens), mais aussi à des formateurs de personnel de maîtrise et d'exécution. A titre indicatif, environ la moitié des sessions inscrites au programme 1990 ont été conduites en anglais, l'autre moitié en français; deux tiers des sessions sont organisées dans des pays en développement d'Afrique, d'Asie et de la Méditerranée. Dans l'organisation des sessions et dans leur contenu technique, le Centre s'efforce de coopérer avec un ensemble d'organisations nationales et régionales touchant au domaine de l'eau. Cette coopération concerne la conception et l'élaboration des programmes, la participation de conférenciers, et l'organisation matérielle pour les sessions tenues dans les pays.

Réunir ainsi, pendant quelques semaines, une quinzaine de professionnels permet notamment aux stagiaires:

- d'enrichir leurs connaissances grâce à l'apport des conférenciers (tous experts de dimension internationale) s'exprimant durant les sessions, et d'ouvrir de nouveaux horizons techniques, financiers, ou concernant la gestion des équipes ;

- d'échanger leurs expériences propres, et ainsi de situer leur niveau technique, l'étendue de leurs problèmes, la dimension de leur fonction, par rapport à ce qui existe dans d'autres pays ;

- de prendre le recul nécessaire par rapport à leur tâches quotidiennes et de faire ainsi progresser leur fonction.

Ce perfectionnement, à travers le dialogue avec des conférenciers de différents horizons (bailleurs de fonds, décideurs en matière de politique de l'eau, gestionnaires, exploitants, ...) donne aux participants des outils pour mieux préparer, mettre en œuvre et gérer les projets et les institutions liés à l'eau. Nombreux aussi sont les participants aux sessions qui viennent y préparer la prise en main d'une nouvelle fonction. Enfin, certaines sessions peuvent être adaptées au cas particulier d'un pays ou d'une organisation nationale, et donc réalisées au niveau national.

\subsubsection{Le conseil en ingénierie de formation}

A côté du programme des sessions internationales, le CEFIGRE prodigue divers services de conseils en ingénierie de formation, à la demande de pays ou d'organisations spécifiques. Il devient instrument d'aide aux mutations des structures nationales du secteur eau. Il a participé notamment à :

- des formations de formateurs ;

- des sessions de formation ou des séminaires nationaux (modification de la Loi sur l'eau, planification, réorganisation technique...);

- la planification des actions de formation, l'évaluation des centres de formation ou la mise en œuvre de projets pédagogiques.

Toutes ces actions associent étroitement éducation et culture. Elles mêlent au souci d'acquisition celui d'utilisation des connaissances, par le biais de l'innovation, dans un environnement permanent de comparaison dans le temps et dans l'espace. Cette formation est associée à des instruments de plus en plus perfectionnés de l'informatique, de la bureautique, des moyens audiovisuels qui modifient profondément les conditions d'émission et de réception des différents messages. Toutes ces évolutions rendent la Formation indissociable de la recherche fondamentale mais aussi de la recherche appliquée et surtout de la recherche pédagogique encore trop méconnue.

\subsubsection{Les technologies nouvelles}

Le CEFIGRE s'informatise et prend une orientation " technologies nouvelles " grâce à la mise en place d'un contrat plan Etat-Région. Les troix axes prioritaires qui vont marquer l'action innovatrice du CEFIGRE au cours des prochaines années sont: l'informatique pédagogique, la vitrine technologique, l'enseignement assisté par ordinateur:

- Le premier axe concerne la mise en place d'une salle informatique dédiée à la pédagogie qui puisse recevoir des techniciens, des ingénieurs et des dirigeants pour une 
formation fondamentale ou continue tant dans le cadre d'une coopération Nord-Sud qu'Est-Ouest.

- Le deuxième axe concerne la mise en valeur des produits innovants. Pour ce faire le CEFIGRE va créer une salle innovation, véritable vitrine technologique, dédiée à l'informatique et aux nouvelles technologies. Cette action constitue le prolongement d'une convention CEFIGRE-Ministère de la Coopération et du Développement dans le cadre de l'élaboration d'un manuel de référence sur les logiciels et les technologies liées à l'eau. - Le troisième axe concerne l'Enseignement Assisté par Ordinateur (EAO). L'EAO est un outil pédagogique qui assure la formation permanente en connaissance générale ou professionnelle. Il permet ainsi aux individus d'assurer leur auto-formation. L'utilisation des moyens audiovisuels de l'informatique et de la bureautique va compléter la panoplie des moyens pédagogiques des enseignements proposés actuellement au CEFIGRE.

Toutes ces actions sont indissociables et tendent vers un seul et même but : la formation à la gestion des ressources en eau. La mise en œuvre du plan informatique conférera au CEFIGRE une dimension unique sur le plan international dans le domaine de l'eau. L'implantation du Centre sur le site de Sophia Antipolis où se trouvent également l'INRIA, l'Ecole des Mines de Paris, le CERAM, le CNRS, le CSTB, les Universités, l'ESSTIN, l'ISI, l'ISIA, etc..., permettra une synergie de dimension unique en Europe en collaboration avec le Centre International de Communication Avancée.

\subsection{Les partenaires du CEFIGRE}

Organisme international de formation à vocation spécifique, le CEFIGRE a développé des actions de partenariat avec l'ensemble de la communauté internationale de l'eau.

\subsubsection{La Coopération avec la Communauté française de l'Eau}

Le CEFIGRE fait bénéficier les pays en développement de l'expertise des pays développés et en particulier de la Communauté française de l'Eau pour :

- la mise en contact de dirigeants du Tiers Monde et d'experts français,

- le développement de la présence française dans des régions à enjeux économiques,

- le lancement d'actions touchant à des technologies nouvelles et créatives,

- des formations coordonnées avec des stratégies de développement des partenaires.

\subsubsection{Les relations avec les coopérations bilatérales}

Le Ministère de la Coopération et du Développement soutient le programme des sessions internationales du CEFIGRE destinées aux pays d'Afrique francophone ainsi que des opérations spécifiques. Le Ministère des Affaires Etrangères a financé un certain nombre de bourses d'études et d'opérations.
La République Fédérale d'Allemagne participe, par le biais de bourses, de missions d'évaluation et d'opérations de formation, aux activités et au développement du Centre. Enfin, le Conseil d'Administration du CEFIGRE désire élargir le caractère européen du Centre.

Les Coopérations scandinaves sont également des partenaires privilégiés du Centre, leurs interventions se basent essentiellement sur le financement d'un certain nombre de bourses et d'opérations. Il s'agit de la SIDA (Swedish International Development Agency), la DANIDA (Danish International Development Agency), la NORAD (Norvegian Royal Administration for Development) et la FINNIDA (Finnish International Development Agency).

\subsubsection{Les relations avec les coopérations multilatérales}

Les relations avec les organismes de coopération multilatérales s'organisent autour des Nations Unies, notamment avec le Programme des Nations Unies pour l'Environnement (PNUE) qui, après avoir joué un rôle déterminant dans la création et le démarrage du CEFIGRE, participe à l'organisation de sessions de formation, à des activités de publications d'ouvrages et au financement de missions d'expertise en matière de formation dans le domaine de l'environnement. Des relations actives sont également maintenues avec le PNUD (Programme des Nations Unies pour le Développement), la Banque Mondiale, l'OMS (l'Organisation Mondiale de la Santé), l'UNICEF (Fonds des Nations Unies pour l'Enfance) et l'UNESCO (Organisation des Nations Unies pour l'Education, la Science et la Culture).

D'autres agences multilatérales participent également aux activités du Centre : parmi celles-ci on peut citer les Banques et Fonds de Développement Régionaux et Interrégionaux: Banque Africaine de Développement, Banque asiatique de Développement, le Fonds International pour le Développement Agricole ; l'Agence de Coopération Culturelle et Technique ; ou encore la Communauté Economique Européenne.

\subsubsection{Répartition des financements du Centre}

Le CEFIGRE est composé d'une équipe de 30 permanents, dont la moitié est constituée de praticiens et de scientifiques des métiers de l'eau. Leurs collègues sont en charge de la gestion et de l'organisation des activités du Centre. Le budget annuel est d'environ quatre millions de dollars par an dont les deux tiers proviennent de fonds internationaux. Les subventions proviennent pour l'essentiel du Ministère de tutelle (Secrétariat d'Etat auprès du Premier Ministre chargé de l'Environnement) et du Ministère de la Coopération et du Développement (fig. 1).

\subsection{La conception des programmes de formation}

L'action du CEFIGRE se déploie en trois temps. Dans le premier, le CEFIGRE identifie en s'inspirant des recommandations de son Conseil Scientifique International et de ses Conseils Scientifiques Régionaux, les problèmes qui se 

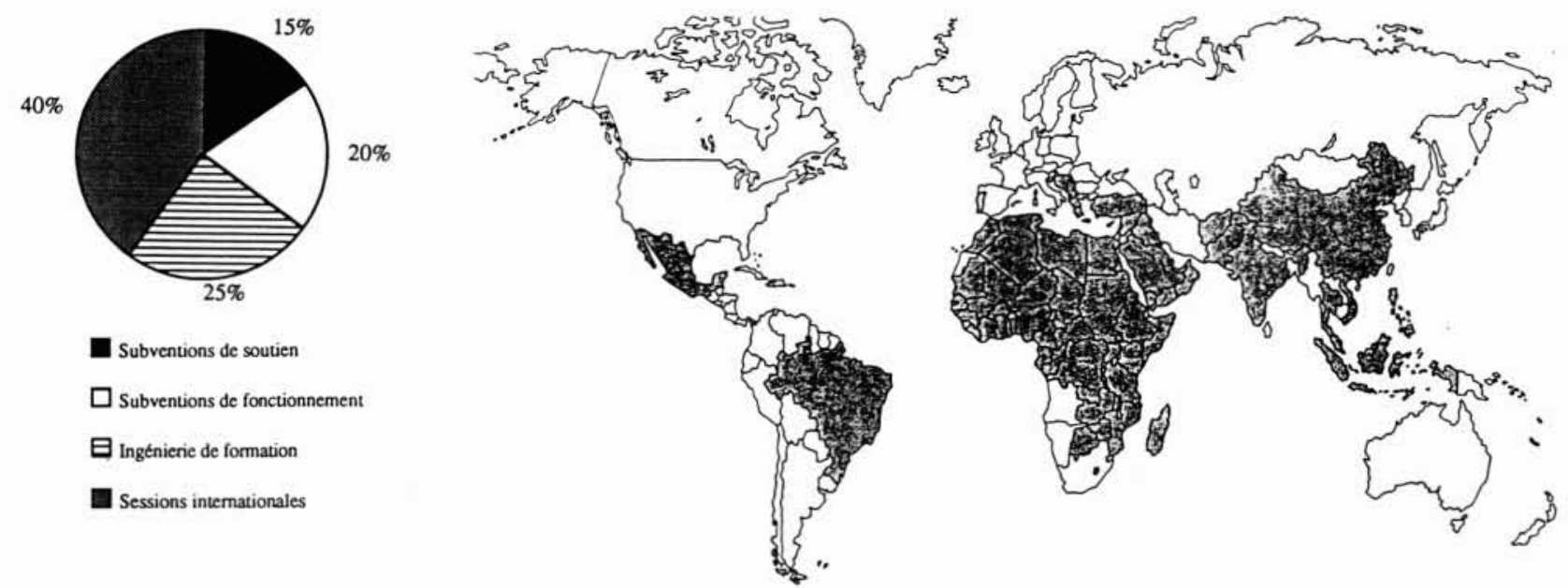

1. Répartition des financements pour l'année 1989.

2. Répartition géographique des activités du CEFIGRE.

posent aux pays en matière d'eau et d'environnement. Ensuite, le CEFIGRE conçoit des actions menant les responsables de l'eau, de l'environnement, de la santé et de l'agriculture à maîtriser les solutions en les diffusant. Enfin, le CEFIGRE procède à une évaluation de la pertinence de ses programmes et de son impact pédagogique.

\subsubsection{Les Conseils Scientifiques}

Les programmes proposés par le Centre résultent des travaux d'un Conseil Scientifique International auquel participent 25 pays en développement et la plupart des agences de coopération. Ce Conseil a été subdivisé en 3 conseils régionaux (Afrique, Asie, Méditerranée et pays Arabes). En 1989, deux réunions régionales du Conseil Scientifique International ont été organisées: l'une à Dakar pour les pays africains, l'autre à Bangkok pour les pays asiatiques. Ces réunions ont permis de mieux adapter les programmes de formation aux problèmes et préoccupations spécifiques des régions.

\subsubsection{Lëlaboration des programmes de formation}

Le programme de chaque session internationale est conçu par l'équipe scientifique du Centre avec l'aide de consultants extérieurs et sous le contrôle de conseillers scientifiques internationaux. Le CEFIGRE fait appel chaque années à plus de 150 confërenciers venant d'une trentaine de pays développés ou en développement et d'organisations internationales. Les sessions s'appuient sur une documentation basée pour une grande part sur les services de l'Association Française pour l'Etude des Eaux (AFEE), premier centre de documentation français en matière d'eau, et dont une antenne est située dans les locaux du CEFIGRE à Sophia Antipolis.

Le CEFIGRE s'efforce d'enrichir régulièrement et systématiquement le contenu de ses sessions de formation, sur la base de suggestions faites à l'occasion de l'évaluation de chaque session par les cadres et dirigeants y ayant participé. Le programme des sessions de formation internatio- nales est articulé autour des quatre thèmes fondamentaux : Ressources en eau, Alimentation en eau et assainissement en milieu urbain, Alimentation en eau et assainissement en milieu rural et l'irrigation, Gestion de l'environnement.

\subsubsection{Lévaluation des programmes}

A l'occasion de chaque session internationale de formation une évaluation du contenu de la session est réalisée. Elle touche à l'organisation matérielle, au contenu pédagogique, à la pédagogie des enseignants, à la durée et la pertinence de chaque thème, au profit qu'en retirent les participants, etc. Ces questionnaires sont alors dépouillés et un rapport d'évaluation est réalisé. Il est adressé à l'ensemble des stagiaires, aux conférenciers et aux organismes de financement. Enfin, ces évaluations permettent de cibler au mieux les thèmes porteurs et les conférenciers les mieux perçus.

\section{Bilan et perspectives}

La pertinence de la création d'un Centre de formation internationale est indiscutable. Aussi nous proposons-nous de présenter, à travers divers descripteurs, l'importance des actions menées et de ses réalisations. Nous présentons l'action géographique, à travers la répartition de ses actions, l'origine de ses conférenciers et des participants ; l'adéquation de son programme à travers l'évolution des secteurs d'activité et, enfin, son évolution, par le biais de la participation aux sessions internationales de formation. Enfin, nous envisageons le cas particulier des ingénieurs hydrauliciens.

\subsection{L'action géographique}

Le CEFIGRE a formé, recyclé, perfectionné environ 3000 cadres et dirigeants de 100 pays d'Afrique, d'Asie, du Moyen-Orient, d'Amérique Latine et d'Europe (fig. 2). 


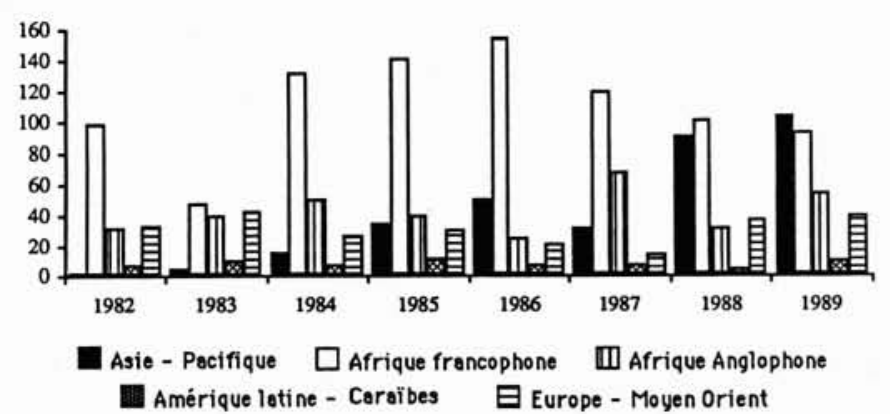

3. Evolution des participants aux sessions internationales de 1982 à 1989 par grandes zones géographiques.

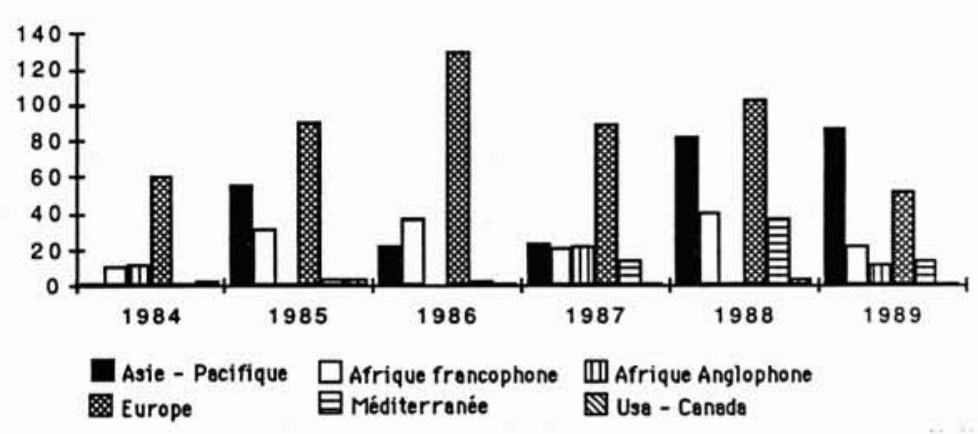

4. Evolution de l'origine des conférenciers aux sessions internationales de 1984 à 1989 par grandes zones géographiques.

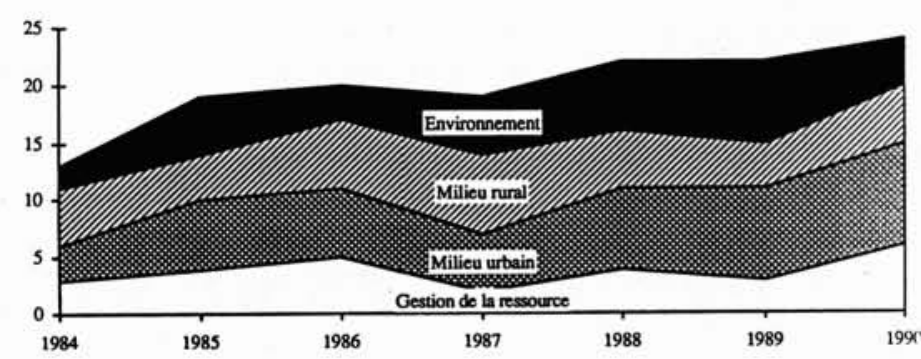

5. Evolution du thème des sessions internationales de formation de 1984 à 1989.

\subsubsection{Les participants}

Les participants aux sessions internationales de formation ont été regroupés en grandes zones géographiques. L'étude de la figure 3 montre la nette progression de la participation de l'Asie, à la suite de la création du centre de Bangkok en 1987. L'Afrique francophone a toujours été un partenaire clef du Centre malgré un certain tassement depuis 1986. Les trois autres zones géographiques sont restées relativement constantes. Le Moyen-Orient (englobant les pays méditerranéens) affirme sa légitimité et la création d'une division est désormais justifiée.

\subsubsection{Les conférenciers}

La figure 4 montre une forte proportion de conférenciers européens et asiatiques, le bloc nord-américain et l'Afrique anglophone restent très peu représentés, alors que l'Afrique francophone et les pays méditerranéens sont en position intermédiaire.

Le tableau ci-dessous montre que l'indicateur " origine des conférenciers " s'est inversé entre 1982 et 1989. Il y a, depuis 1988, une majorité de conférenciers issue de pays en développement. Cet élément est tout à fait en adéquation avec la "politique » du Centre qui est de donner largement la parole aux conférenciers issus des pays en développement.

\subsection{Les secteurs d'activité et leur évolution}

La figure 5 montre que la proportion des quatre grands thèmes a été préservée dans le temps. Une étude plus poussée des sous-thèmes montre l'adéquation des sujets proposés avec la problématique des pays en développement. Le milieu urbain semble cependant prendre une extension supérieure aux autres ces dernières années.

Une tendance, développée depuis quelques années vise à la réalisation de synthèses méthodologiques thématiques et à la publication de manuels didactiques sur des sujets spécifiques du domaine de la gestion des ressources en eau. Cette approche permet de mieux faire connaître le savoirfaire du CEFIGRE.

Tableau 1. Evolution des conférences aux sessions internationales de formation de 1982 à 1989.

\begin{tabular}{|c|c|c|}
\hline Origine des conférenciers & $\begin{array}{c}\text { Pays en voie de } \\
\text { développement }\end{array}$ & $\begin{array}{c}\text { Pays } \\
\text { industrialisés }\end{array}$ \\
\hline $1982: 88$ conférenciers & $28 \%$ & $72 \%$ \\
$1983: 58$ conférenciers & $17 \%$ & $83 \%$ \\
$1984: 91$ conférenciers & $30 \%$ & $70 \%$ \\
$1985: 188$ conférenciers & $50 \%$ & $50 \%$ \\
$1986: 194$ conférenciers & $33 \%$ & $67 \%$ \\
$1987: 172$ conférenciers & $47 \%$ & $43 \%$ \\
$1988: 268$ conférenciers & $60 \%$ & $28 \%$ \\
\hline
\end{tabular}




\subsection{La participation aux sessions internationales de forma- tion}

La participation aux sessions internationales de formation semble être un moyen d'appréhender le niveau de performance du Centre. On note qu'entre 1982 et 1989 le nombre de semaines stagiaires a été multiplié par deux et que le nombre de stagiaires a été multiplié par trois durant la même période.

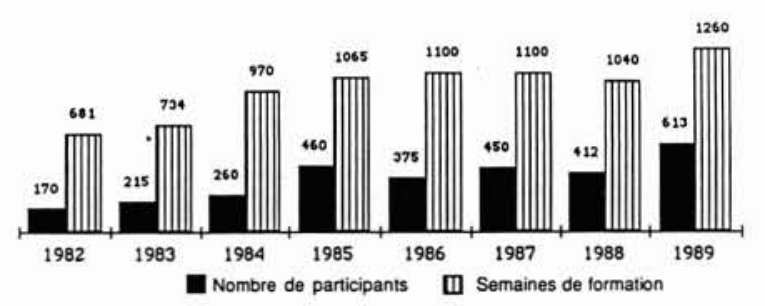

6. Evolution du nombre de stagiaires et du nombre de semaines de formation de 1982 à 1989.

\subsection{La place des ingénieurs hydrauliciens}

L'analyse de la base de donnée "participantsconférenciers" du CEFIGRE ne permet pas de déceler facilement le niveau de participation des ingénieurs hydrauliciens. En effet, on en dénombre une centaine, dont environ $30 \%$ sont intervenus en qualité de conférencier, cependant on perd rapidement leur trace en raison de leurs évolutions de carrière. Dans les pays en développement la promotion est relativement rapide et amène les cadres compétents à passer après quelques années d'un poste d'opérationnel à un poste de dirigeant.
La promotion proposée étant essentiellement à caractère "administratif» la nécessité de former des ingénieurs opérationnels reste donc permanente. Cette remarque met en évidence la nécessité d'une formation continue encore plus importante que dans les pays industrialisés.

Les ingénieurs hydrauliciens qui sont venus au CEFIGRE ont reçu une bonne formation initiale ce qui leur permet d'évoluer rapidement. D'une manière générale on dénombre essentiellement des ingénieurs de fabrication ou de conception mais peu d'ingénieurs de recherche. Ils ont une bonne formation scientifique de base notamment en mécanique des fluides et en calcul numérique. L'informatique reste souvent embryonnaire et une formation complémentaire à "l'informatique de l'ingénieur est une des recommandations que l'on peut formuler en matière de formation continue pour les années à venir. La formation continue doit avoir lieu in situ et être orientée sur des expériences concrètes et des échanges d'expériences entre les différents participants. Enfin, elle doit être réalisée par des ingénieurs des pays en développement, la problématique n'étant plus d'ordre fondamentale mais opérationnelle et spécifiquement orientée sur des expériences vécues dans les pays.

\section{Conclusion}

Comme nous l'avons vu les enjeux sont planétaires. Dans cette optique le problème n'est plus d'analyser des modèles anciens mais de comprendre comment la formation peut apporter sa contribution pour trouver des réponses aux questions que les peuples se posent. C'est l'approche qu'a retenu le CEFIGRE à travers son intégration dans quelques 80 pays du tiers monde.

Il est certain qu'une gestion consciente et intelligente des ressources en eau est un grand défi à l'aube du $\mathrm{XxI}^{\mathrm{e}}$ siècle. L'humanité doit y faire face et la formation en est le point de passage incontournable.

\section{Bibliographie}

[1] Pour la Science. Numéro spécial de novembre 1989, n 145.

[2] La Recherche. Numéro spécial l'eau. Mai 1990, n²21.

[3] Conférence des lauréats du prix Nobel. Promesses et menaces à l'aube du XXf siècle. Edition O. Jacob, 1988, Paris.

[4] Detay M. et Poyet P., 1990. Introduction aux méthodes modernes de maîtrise de l'eau. Hydrogéologie, $\mathrm{n}^{\circ} 1,1990$, pp. 3-25, 9 fig., 3 tabl.

[5] United Nations, 1977. Desertification: its causes and consequences. Compiled and edited by the Secretariat of the United Nations Conference on Desertification, Nairobi. Pergamon Press, $448 \mathrm{p}$. 